A N N A L E S Annales de Bretagne et des Pays de l'Ouest

\title{
La recherche à l'université du Maine (Le Mans) de 2000 à 2007
}

Liste des thèses et mémoires soutenus et déposés à l'université du Maine

\section{OpenEdition \\ Journals}

Édition électronique

URL : http://journals.openedition.org/abpo/250

DOI : $10.4000 / a b p o .250$

ISBN : 978-2-7535-1512-3

ISSN : 2108-6443

Éditeur

Presses universitaires de Rennes

Édition imprimée

Date de publication : 31 décembre 2008

Pagination : $321-326$

ISBN : 978-2-7535-0808-8

ISSN : 0399-0826

Référence électronique

"La recherche à l'université du Maine (Le Mans) de 2000 à 2007 ", Annales de Bretagne et des Pays de l'Ouest [En ligne], 115-4 | 2008, mis en ligne le 31 décembre 2008, consulté le 15 septembre 2020

URL : http://journals.openedition.org/abpo/250

Ce document a été généré automatiquement le 15 septembre 2020.

(c) Presses universitaires de Rennes 


\section{La recherche à l'université du Maine (Le Mans) de 2000 à 2007}

Liste des thèses et mémoires soutenus et déposés à l'université du Maine

1 Précédente publication : ABPO 111-2 (2004), période 1998-2003.

\section{BIBLIOGRAPHIE}

\section{Master 1}

\section{4}

AlloteAu, Marie, Les églises du canton de La Loupe (Eure et Loir) au xixe siècle, dir. B. WACHÉ.

AUBRY, Aurore, Le théâtre de Mamers (1851-1914), dir. B. WACHÉ.

CLEROT, Anaïs-Marie, Un consul de France au Maroc : Jean-Baptiste Estelle (1690-1699), dir. H. DAussY.

BAHJAOUI, Laïla, La marche à l'indépendance du Maroc : regards croisés de la presse mai 1953-mars 1956, dir. C. MANIGAND.

BRUNEAU, Amélie, Presse et opinion face aux évènements d'Indochine (mai 1953-frévrier 1955), dir.

C. MANIGAND.

CALLU, Sabrina, La politique agricole commune dans le canton de La Ferté- Bernard. 1984-2003, dir. N. VIVIER.

CORNU, Joël, Les monnaies du Maine : organisation et circulation monétaire au Moyen-Age en France (VIeXIVe siècle), dir. A. RENOUX. 
CLOLUS, Nicolas, Répression et conversion : la politique de l'intendant Lallemant de Lévignen envers les protestants de la généralité d'Alençon (1726-1766), dir. D. BoIssoN.

DENEUIL, Virginie, Le Nord Sarthe à l'époque gallo-romaine : nature de l'occupation humaine, dir. E. BERTRAND.

DUPIN, Sophie, Les chapelles castrales dans le Haut-Maine à l'époque moderne, dir. L. BOURQUIN.

EVRARD, Gaëlle, Les inondations au Mans au XXe siècle, dir. N. VIVIER.

FonTAINE, Aurélie, Les cabaretiers au XVIIIe siècle : l'exemple de Malicorne, dir.F. PITOU.

GARNIER, Sandrine, Mottes castrales et manoirs dans le canton de Mamers (1000-1520), dir. A. RENOUX. GoszTolA, Anne, La prostitution et la police des moeurs au Mans au XVIIIe siècle, dir. L. BOURQUIN.

GUERIN, Cécile, Les envois de peintures par l'État au musée du Mans entre 1799 et 1929, dir. B. WACHÉ et F. LUCBERT.

LePAReUR, Camille, Déclin du Paganisme et sanctuaires en Occident à la fin de l'Antiquité,(IVe-VIe siècle),dir. M.C. L'HUILLIER.

LESBAUDY, Marie-Caroline, La restauration des édifices religieux dans la Sarthe. Son évolution du XIXe siècle au xxe siècle, dir. B. WACHE.

LHERMITTE, Benoît, Le bâti civil de la ville sanctuaire du Vieil-Evreux (27) à l'époque gallo-romaine, dir. E. BERTRAND.

MEGDOUL, Farid, Santé, maladie et médecin dans le monde romain : l'apport des correspondances (IVe siècle), dir. M. C. L'HuILLIER.

MENARD, Céline, La communauté protestante de Bellème au XVIIe siècle, dir. D. BoIsson.

MIGNOT, Yanick, Le village du Haut-Maine à la fin du XVIIIe siècle : composition et morphologie à partir du premier cadastre, dir. F. PIтоU.

MOREAU, Hélène, Les Mariandyniens d'Héracléé du Pont : entre esclavage et liberté, dir. A. AvRAM.

OLIVIER, Nicolas, Ravitaillement alimentaire et marché noir - Alençon - 1940-1949, dir. B. WACHE.

OUDARD, Amandine, La protection des Monuments Historiques - Le Mans au XIXe siècle, dir. B. WACHE.

PEUVION, Cyrille, Inventaire des mottes, hébergements, maisons fortes et manoirs du canton du Mans : XIeXve siècle, dir. A. RENOUX.

PlUQUET, Magali, La rue du petit pont neuf au Mans à la fin du XVIIe siècle, dir. F. PITOU.

Poulain, Tony, Le butin de guerre sous la République romaine, dir. A. AlLELY.

RADENAC, Guillaume, L'image du dépoté du travail dans l'opinion française depuis les premières lois relatives à la main-d'oeuvre jusqu'à nos jours, dir. C. MANIGAND.

RENARD, Arnaud, Origines et enjeux de la mise en place d'un réseau de communication dans le processus de modernisation et d'ouverture des Highlands. xviiie-xixe siècle, dir. N. VIVIER.

RENAUDIN Hélène, Les manoirs ruraux et leurs seigneurs dans les cantons de Beaumont sur Sarthe, Conlie et Sillé-le-Guillaume (1450-1520), dir. A. RENOUX.

SALLARD, Carinne, Les manoirs de 1450 à 1520 dans les cantons d'Ambrières, Gorron et Landivy, dir. A.

Rennoux

SOULADIE, Jean-Jérôme, L'Esclavage et la dépendance dans la métamorphose d'Apulée, dir. M. C. L'HUILLIER. 
TERTRE, Adeline, Les foyers de Mamers au XVIIIe siècle, dir. J.-M. CONSTANT.

VenI, Jonathan, Le Parti Communiste dans la Sarthe 1919-1939, dir. P. FouCAULT.

WEISSE, Marie, La vie musicale au Mans pendant l'entre-deux guerres, dir. B. WACHE.

\section{5}

BEAUMONT, Claire, La justice de paix dans le canton de Montmirail au XIXe siècle, dir. N. VIVIER.

BEN-DRISS, Hajer, L'économie sarthoise 1845-1860, dir. N. VIVIER.

BESNARD, Florian, L'approvisionnement et l 'alimentation de la Maison Dieu de Coeffort au Mans entre 1450 et 1510 , dir. A. RENOUX.

BIREE Patrick, Loisirs et distractions au Château de Carrouges aux XVIIIe et XIXe siècles, dir. S. GRANGER.

BOUFFARD, Gaëlle, La femme romaine dans les œuvres de Plaute et de Térence aux iiie et iie siècle av. J.-C., dir. A. ALLELY.

BOUTIER, Sonia, L'instrumentum domesticum en territoire cénoman, dir. E. BERTRAND.

BOULAY, Julien, L'électrification de la ligne Paris-Le Mans 1936, dir. N. VIVIER.

BUISSON, Julien, Les élections présidentielles de 1887, 1894 et 1895, dir. N. VIVIER.

COLLIN, Vivien, La construction européenne vue à travers la presse locale, 1945-1992, dir. N. VIVIER.

DAVAZE, Virginie, Les institutions de Mégare et de ses colonies, dir. A. AVRAM.

EDON, Carole, Les vacances scolaires des petits manceaux dans l'Entre deux-guerres, dir. B. WACHÉ.

GAUTUN, Nicolas, L'invasion prussienne dans l'Orne (1870-1871). Déroulement et impact sur la population, dir. N. VIVIER.

GELE, Marie, L'Orne combattante d'Alençon, dir. B. WACHÉ.

GIRARD, Stéphanie, Les petites églises rurales des doyennés de Fresnay-sur-Sarthe et de Lignières-la-Corelle au Moyen-Age, dir. A. RENOUX.

GROUAS, Estelle, L'image du roi à travers l'Histoire Universelle de Jacques-Auguste de Thou (1515-1589), dir. H. DAUSSY.

HETTIER, Pascale, Les transformations urbaines du Mans sous le Second Empire, dir. N. VIVIER.

HUMBERT, Nolween, L'image des femmes dans la publicité, dir. N. VIVIER.

JUIGNER, Xavier, L'abbé Anjot, historien de la Mayenne. Un prêtre érudit de la Belle-Epoque entre apologistique et histoire critique, dir. B. WACHÉ.

LOISEAU, Yoann (né le 6 mai 1983), L'agriculture galicienne face à l'intégration dans le marché commun, dir. N. VIVIER.

LOISEAU, Yoann (né 9 novembre 1982), L'Abbaye de Saint Calais au XVe siècle, dir. A. RENOUX. MEKDOUD, Nouara, Les écoles primaires catholiques au Mans entre 1880 et 1910, dir. B. WACHÉ. Nion, Ludivine, L'Ami des campagnes : histoire d'un journal d'information et d'éducation destiné aux villageois, dir. L. BOURQUIN.

PERON, Christina, Le discours médical autour du thé, café, chocolat du XVIIe au XIXe siècle, dir. L. BOURQUIN. 
PICHARD, Edwige, Le prieuré de Tuffé au temps des Mauristes (1646-1766), dir. D. BoIsson.

PINAUD, François, Les mottes castrales et maisons fortes dans le canton de Sablé-sur Sarthe au Moyen-Age, dir. A. RENOUX.

PINEAU, Nicolas, Les manoirs des cantons d'Ecommoy et du Grand-Lucé au Moyen-Age. A. RENOUX.

PIPELIER, Nicolas, L'Institution Notre Dame de Sainte Croix au Mans de 1830 à 1869, dir. B. WACHÉ.

REMARD, Marie-Anne, Le collège de la Flèche en 1771, dir. D. BOISSON.

ROULIER, Cécile, Les transformations de la Ville du Mans : le Tunnel et les quais, rive gauche 1848-1877, dir. N. VIVIER.

SAILLARD, Sandrine, Mémoire d'un officier sur la campagne d'Espagne (1808-1814): Jean-Baptiste Dauvais, Relation de captivité en Espagne, 1824, dir. L. BOURQUIN.

SAULIN, Gaëlle, Le collège de l'Oratoire du Mans et ses élèves (1660-1792), dir. D. BOISSON.

souton, Baptiste, L'idée de coexistence pacifique à travers l'Histoire Universelle de Jacques-Auguste de Thou, dir. H. DAUSSY.

TAURAN, Audrey, Les ambassades mémorables vers les Empereurs du Japon d'Arnoldus Montanus, dir. H. DAUSSY.

THIROUARD, Sébastien, La JAC dans le Perche pendant les années 1930-1950, dir. B. WACHÉ.

TOUZE, Hélène, Enseignement, Oppositions et Résistance sous le régime de Vichy 1940-1944, dir. B. WACHÉ. VANTARD, Amélie, Les confréries du Rosaire dans le diocèse de Sees : xviie-xviiiesiècles, dir. F. LUCBERT et D. BOISSON.

WIMART, Julie, La protection des monuments historiques au xixe siècle dans la Sarthe, dir. B. WACHÉ.

\section{6}

AUDEBERT, Marine, Recherches sur la « Poliorcétique » d'Enée le Tacticien, dir. A. AVRAM.

BONNEFOY, Elodie, Étude du quartier Saint Nicolas au Mans au xviiie siècle, dir. L. BOURQUIN.

BRIAND, Séverine, Le comice agricole de Mamers, 1848-1868, dir. N. VIVIER.

CHABRUN, Vanessa, La construction des mairies du canton de Malicorne XIXe-XXe siècles, dir. N. Vivier.

CHAUVEAU, Florence, L'accès aux soins médicaux de la population indigente 1855-1898 : l'exemple du Mans, dir. N. VIVIER et H. GUILLEMAIN.

COULANGE, Yohann, Les Immigrants du Maine en Nouvelle France et particulièrement à Montréal :

1637-1773, dir. F. PITOU.

COUTAUD, Ghislain, L'Hôtel Dieu de Coëffort : l'implantation des prêtres de la Mission (lazaristes) en 1645, dir. F. PITOU.

DONIES, Delphine, Les mémoires de Michel de Castelnau : étude d'un discours sur les guerres de Religion et sur l'état de l'Angleterre (1559-1570), dir. H. DAUssY.

DONNET, Guillaume, Le culte de Dionysos à Milet et ses colonies, dir. A. AVRAM

DROU, Hélène, La criminalité dans la Sénéchaussée du Maine en 1769, dir. L. BOURQUIN.

FOURNIER, Fabien, L'exposition régionale du Mans en 1880, dir. N. VIVIER. 
GASNIER, Lauréanne, L'hôtel Amellon de Saint Cher, de la construction à la rénovation, dir. S. GRANGER. GUICHEMER, Carole, Un bourgeois gentilhomme au xviiie siècle : Gabriel Chenon du Boullay, dir. L. BOURQUIN.

GRUDE, David, Les magistrats du Présidial du Mans au xviiie siècle, identité prépondérance d'une élite judiciaire et urbaine, dir. F. PITOU.

JAFFRES, Loïc, Le rôle de Michel de La Huguerie au service des princes protestants (1570-1581), dir. H. DAUSSY.

JOLLY, Gwenaëlle, Les Sarthois face à l'électrification rurale vers 1920-1975, dir. N. VIVIER.

LASCAUX, Thomas, Alcoolisme antialcoolisme en Sarthe au xixe siècle, dir. N. VIVIER et I. JABLONKA LEBORGNE, Patrick, Les citées grecques de la Mer Noire sous la menace des barbares, dir. A. AVRAM. LEDAUPHIN, Anthony, Les figurines en terre cuites d'époque gallo-romaine dans les Cités des Aulerques Diablintes et Aulerques Cénomans, dir. E. BERTRAND

LEMETAYER, Elodie, L'artisanat de la céramique dans la vallée de la Jousse, des fondements anciens à la valorisation culturelle, touristique et économique de demain, dir. F. PITOU.

LEPROUT, Marina, Le retour de prisonniers de guerre sarthois après la Libération de la France, dir. B. WACHE et S. TISON.

LENNE, Julien, Les frères Wright au Mans, dir. N. VIVIER et S. TISON.

MERE, Marie-Adeline, A la rencontre de Michel Boyer, une figure littéraire et musicale mancelle méconnue (1768-1858), dir. S. GRANGER.

MEZANGER, Florian, Claude de Mondoucet, résident pour le roi de France aux Pays-Bas : les enjeux de la diplomatie française de 1572 à 1575, dir. H. DAUssY.

MORIN, Matthieu, Un ambassadeur français en Angleterre au xvie siècle : Bertrand de Salignac De La Mothe Fénelon (1568-1575), dir. H. DAussY.

RAQUIDEL, Charlotte, Ambroise II de Loré (vers 1393-1446) et l'assise seigneuriale du lignage, dir. A. RENOUX.

RONDET, Manuela, L'annexion de la commune de Pontlieue et ses conséquences sur la voirie du quartier : 1866-1910, dir. N. VIVIER.

TORCHET, Marie-Maud, Les Mémoires d'Outre-Mer d'Adhémar Leclère : 1886-1911, dir. B. WACHE et S. TISON.

VIROS, Fanny, Yvré-l'Evêque : indices d'urbanité dans un village près du Mans, dir. L. BOURQUIN.

\section{7}

ALLORY, Jessica, Étude des cas de divorces enregistrés dans la ville du Mans entre 1884 et 1894, dir. B. WACHÉ.

BLONDIN, Elodie, Un protestant au service du roi : le Maréchal de La Force (1556-1632), dir. H. DAUssY. BOUDIN, Jérôme, Inventaire des églises du doyenné de Montfort (Moyen Âge), dir. A. RENOuX. BOURRIGAULT, Mathieu, Le rôle politique des esclaves durant les guerres civiles et les conjurations à Rome de 133 à 31 av. J.-C., dir. A. ALLELY 
BRANDO-TALERO, Carolina, Mise en place d'une classe du patrimoine sur les Jardins des Plantes du Mans, dir. S. GRANGER.

BRETEAU, Violaine, La première mission diplomatique de Jacques de Ségin de Pardaillan (1583-1585), dir. H. DAUSSY.

CHARRIOUX, Séverine, Eugène Hucher (1814-1889) et la conservation des monuments historiques du département de la Sarthe, dir. B. WACHÉ.

CHAUVIN, Sébastien, La seigneurie de Foulletourte au Moyen Âge. Essai d'histoire économique et sociale, dir. A. RENOUX.

COGNASSE, Jennifer, Un hôtel particulier des Lumières au XXIe siècle : l'hôtel Nepveu de Rouillon au Mans. Construction, restauration, réhabilitation, dir. S. GRANGER.

COLLoT, Cécile, La fonderie de Saint Pavin-industrie et patrimoine, dir. N. VIVIER.

DAME, Clément, Conception d'une visite muséographique à destination d'un public de cycle 3 ou la vie quotidienne sous l'Ancien Régime, dir. F. PITOU.

DE CASTRO, Adeline, Les décors intérieurs des églises prémontrées de l'Orne : l'influence de la réforme de Lorraine, dir. D. BOIsson.

DOUSSET, Flore, Les implantations humaines au bord des rivières et les conflits liés à l'eau, à Mamers l'exemple du Conté du Maine dans la seconde moitié du XVIIIe siècle, dir. F. PITOU.

DUPRE, Julien, Les actionnaires de la comédie, culture et sociabilité des élites au Mans à la fin du XVIIIe siècle, dir. S. GRANGER.

GAUGIN, Étienne, La place de la République au Mans-1880-1939, dir. N. VIVIER.

GAVALET-CANTIN, Camille, La bibliothèque de l'Abbaye Saint Vincent du Mans au xviiie siècle, dir. S. GRANGER.

GENDRONNEAU, Amandine, Manoirs des cantons de Saint-Paterne, Mamers et La Fresnay-sur-Chédonet (xiiie-xvie siècle), dir. A. RENOUX.

GUILLEMIN, Thomas, Isaac Papin : réflexion sur la tolérance et la conversion d'un théologien protestant dans la République des Lettres, dir. D. BoIsson.

HEITZ, Stéphanie, Le Mans, entre rivière et murailles : le quartier des Tanneries au XVIIIe siècle, dir. S. GRANGER.

JANVIER, Laurie-Anne, L'exaltation du pouvoir royal à l'époque de Louis XIII, dir. L. BOURQUIN.

LAUNAY, Vanessa, L'action des notables dans la municipalité de Bonnétable, dir. N. VIVIER.

LE GALL, Mélanie, L'enseignement primaire public dans les écoles de garçons (Fâtines, Montfort-le-Retrou et Parigné-l'Evêque), dir. B. WACHÉ.

LELIEVRE, Ludovic, Le comice agricole de Mortagne au Perche, dir. N. VIVIER.

LOUIS, Dorothée, Archives municipales et communautaires du Mans. Traitement et indexation d'un fond iconographique, dir. S. GRANGER.

MAZAUD, Valérie épouse PROST, La guerre scolaire à propos de l'enseignement secondaire féminin, dir. B. WACHÉ.

MEUNIER, Julie, Jean Pissot Desperrières, notable de La Bazoge dans la deuxième moitié du XVIIIe siècle, dir. L. BOURQUIN. 
MORIN, Linda, L'image d'Henri IV d'après l'historiographie du XVIIIe siècle, L. BOURQUIN. RENOU, Orlane, Les châtiments des crimes dans la Sénéchaussée du Maine et siège présidial du Mans (1714-1791), dir. F. PITOU.

RENOU, Anthony, L'image d'Henri IV à travers les mémoires de Sully, dir. C. MICHON. ROUSSEAU, Virginie, Le Prieuré de Vivoin, Action éducative au Patrimoine, dir. F. PITOU. SIMONEAU, Hugues, Manoirs des cantons de Mayet et Pontvallain au Moyen Âge, dir. A. RENOUX. TERTRAIN, Romain, Valorisation des devantures et enseignes commerciales, dir. S. GRANGER.

VALENTIN, Julia, L'inventaire de flacons de matières premières datant de la période coloniale. Muséum d'histoire naturelle de Marseille, dir. N. VIVIER.

\section{Master 2}

\section{4}

D'IRUMBERRY DE SALABERRY, Claire, Le style « Louis XIII provincial dans l'architecture des châteaux du Maine et de l'Anjou », dir. J.-M. CONSTANT et L. BOURQUIN.

DuchEMIN, Amélie, La question protestante sous le règne de Louis XV dans l'optique gouvernementale (1723-1774), dir. D. BoIsson et F. PITOU.

GAIGNARD, Michel, Démocrates chrétiens en Sarthe, Mayenne, Maine-et-Loir entre 1925 et 1965, Dir.

C. MANIGAND et N. VIVIER.

GoRIN, Stéphane, Missions catholiques française, protectorat religieux français et chrétiens européens au Moyen-Orient, Dir. C. MANIGAND et B. WACHÉ.

LEMAITRE, Sophie, La Sarthe, l'aventure du premier quotidien sarthois sous la IIIe République, Dir.

C. MANIGAND et B. WACHÉ.

LEMIERRE, François-David, Jean-Jacques Servan Schreiber, un journaliste engagé en politique, Dir.

C. MANIGAND et N. VIVIER.

LENORMAND, Dominique, Un curé parmi les notables de Craon dans le Haut Anjou à la veille de la Révolution, dir. D. BoIsson et F. PITOU.

MARTIN VAN DER HAGEN, Guillaume, Autorité et hiérarchie dans le monde enseignant au XIXe siècle, dir. S. ETUL et N. VIVIER.

PAPIN, Marie-Noëlle, L'intégration des Sarthois dans l'effort de guerre du IIIe Reich, Dir. N. VIVIER et B. WACHÉ.

PINHEIRo, Julia, Imprimeurs et libraires à Saumur, La Flèche, Le Mans et Alençon au XVIIe siècle, dir. D. BOISSON.

2005

BOURGAULT, Ludovic, Théâtre et amphithéâtres en Troisième Lyonnaise, dir. R. SOUSSIGNAN et E. BERTRAND. 
BRUNEAU, Amélie, La bataille de Dien Bien Phu dans la mémoire collective (1954-2004). Une intégration difficile, dir. N. VIVIER et B. WACHÉ.

CORNu, Joël, Circulation du Mansois dans l'Ouest de la France médiévale (XIe-XIVe siècle), dir. A. RENoux et M. BOMPAIRE.

DENIEUL, Virginie, Le Nord Sarthe à l'époque gallo-romaine : les modalités de l'implantation humaine, dir. R. SOUSSIGNAN et E. BERTRAND.

MignoT, Yanick, La cartographie à l'époque moderne, dir. F. PITOU.

PAVE, François, Le journal de Jules Bedeau ; un artilleur dans la chine des boxers, dir. P. FouCAULT et N. VIVIER.

RENAUDIN, Hélène, Les manoirs ruraux et leurs seigneurs dans les cantons de La Flèche et de Malicorne à la fin du Moyen Âge (1450-1520), dir. A. RENOuX et P. RAVINET.

TESTART, Jean-Benoît, L'image de la Prusse et de Frédéric II dans la littérature des Lumières, dir.

L. BOURQUIN et F. PITOU.

WEISSE, Marie, La société chorale du Mans de 1857 à 1935, dir. B. WACHÉ.

\section{6}

BEAUMONT, Claire, La justice de paix dans le canton de Montmirail au XIXe siècle, dir. I. JABLONKA et N. VIVIER.

COLLIN, Vivien, La construction européenne vue à travers la presse locale 1957-1992, dir. N. VIVIER et B. WACHÉ.

CHOLMLET, Mathilde, Les écrits du For privé dans le Haut Maine à l'époque moderne (1490-1865), dir. S. GRANGER et F. PITOU.

DAVAZE, Virginie, Les institutions à Mégare et dans ses colonies, dir. A. AVRAM et J.-Y. STRASSER. GROUAS, Estelle, La construction du mythe du sauveur et de l'homme providentiel dans la légende henricienne à travers les écrits de la fin du XVIe et du début du XVIIe siècle, dir. L. BOURQUIN et H. DAUSSY. HUMBERT, Nolwenn, L'évolution des femmes en France de 1945 à nos jours à travers Elle et Marie Claire, dir. N. VIVIER et B. WACHE.

LOISEAU, Yoann, L'irrigation en Idaho de 1870 à nos jours, dir. N. VIVIER et B. WACHÉ.

PINAUD, François, Inventaires des mottes et des maisons fortes dans le canton de Ballon (Xe-Xve siècle), dir. A. RENOUX et . BRUAND.

VANTARD, Amélie, « Ecce, Mitte » les jésuites français et le désir des Indes aux XVIIe et XVIIIe siècles, dir. F. PITOU et D. BoIsSon.

2007

BOUTIER, Sonia, L'instrumentum domesticum dans le territoire des Aulerques Cénomans, dir. E. BERTRAND et R. SousSIGNAN.

BRIAND, Séverine, Le comice agricole du canton de Mamers 1848-1929, dir. N. VIVIER et B. WACHÉ. 
CHABRUN, Vanessa, Étude d'un bâtiment municipal : la mairie à travers 2 cantons de la Sarthe : Château du Loir et Malicorne XIXe-XXe, dir. N. VIVIER et B. WACHÉ.

CHAUVEAU, Florence, L'assistance médicale gratuite en Sarthe 1902-1939, dir. H. GUILLEMAIN et N. VIVIER. GUILLARD, Baptiste, La perception de l'histoire en fonction des sensibilités politiques, dir. N. VIVIER et B. WACHÉ.

LE BORGNE, Patrick, Cités grecques pontiques et propontiques sous la menace barbare durant l'époque hellénistique, dir. A. AvRAm et R. SoussignAN.

LENNE, Julien, L'aviateur Wilbur Wright au Mans, juin 1908-janvier 1909, dir. S. TISON et N. VIVIER. LoISEAU, Yoann, Les seigneuries du Lucé et de Pruillé-l'Eguiller à la fin du Moyen Âge, dir. A. RENoux et . BRUAND.

MEZANGER, Florian, Francis Walsingham, ambassadeur d'Elisabeth Iere à la cour de France, dir. F. PITOU et H. DAUSSY.

TECHATAM, Suangsuda, Le Siam au XVIIe siècle dans le récit de Jacques de Bourgeois, un missionnaire français des MEP, dir. L. BOURQUIN et F. PITOU.

\section{Thèses}

\section{4}

BERTIAUX, M.-C., Les résidences des évêques de Troyes dans leur diocèse à la fin du Moyen Âge, dir. $\mathrm{P}$. CONTAMINE.

LEANDRO DE MEDEIROS, Ricardo, Feuilleton de radio et publicité : la mémoire de la réception à Florianopolis pendant les années 1960, dir. B. WACHÉ.

Michon, Cédric, Le prélat d'État sous François 1er et Henri VIII, dir. J. M. CONSTANT.

TRIMOREAU, Thierry, Les prêtres réfractaires pendant la Révolution française : l'exemple du Haut Maine, dir. J.-M. CONSTANT et M. MENARD.

\section{5}

DEBRAY, Catherine, L'image de la ville de Gaule dans les sources littéraires latines (284-483) : Terminologie de la ville et de ses composantes architecturales, dir. J. BIARNE.

LEVILLAIN-ANGoulVANT, F., Le théâtre religieux dans le Maine à la fin du Moyen Âge (1450-1550), dir. H. MARTIN.

LIGNE, André, Naissance et évolution d'une congrégation religieuse : les soeurs Marianites de Saint Croix du Mans, dir. B. WACHÉ.

\section{6}

BOUTEILLER, Xavier, Le territoire de Corinthe : transformations politiques et aménagements du paysage (440 av. J.-C. - 96 apr. J.-C.), dir. R. SoussignAN.

HUBERT, Benoît, Mémoires de Jean Baptiste Leprince d'Ardenay. Approche d'un notable manceau au siècle des Lumières, dir. A. FILLON. 
LIGNEREUX, Aurélien, Force à la loi ? Rébellions à la gendarmerie et autorité de l'État en France (1800-1859), dir. N. VIVIER.

MAZURIER, S., Résidences aristocratiques fossoyées et pouvoirs dans la baronnie de Laval (XIe-XVe siècle), dir. D. BARTHÉLEMY.

\section{7}

DE LAPEYRIERE, Marie-José, Le mouvement théosophique en France (1876-1919), dir. B. WACHÉ.

ZANNIER, Marie-Pierre, Paysages du grand domaine et normes agronomiques de Caton à Pline l'Ancien, dir. R. SOUSSIGNAN. 